UDC

\title{
Nonlinear electrical properties of nanostructured porous silicon films
}

\author{
Zhanabaev Z.Zh., Ibraimov M.K., Sagidolda Y. \\ Al-Farabi Kazakh National University \\ Al-Farabi avenue 71, Almaty, 050038, Kazakhstan \\ erulan.s@yandex.ru
}

\begin{abstract}
Porous silicon films have been prepared by electrochemical etching. Morphology of the films has beenstudied by scanning probe microscope characterized by presence of self-similar structures with different scales from hundreds of nanometers to microns. Current-Voltage characteristic of nanofilms has several minima in contrast to the well-known effect of negative resistance in tunnel diodes. For theoretical description of the experimental results we suggest a new concept of "non-linear fractal".
\end{abstract}

Keywords: porous silicon, nanofilms, negative resistance, tunneling effect, non-linear fractal.

\section{Introduction}

Porous silicon (Por-Si) is a perspective material of nanoelectronics, photonics, optoelectronics and other areas of new technologies [1-8]. It was accidentally discovered by A. Uhlir [9] who investigated the process of electrochemical polishing of crystalline silicon in solutions containing hydrofluoric acid. Although the Por-Si has been opened at the end of 50 years of XX century, a substantial interest in him was caused by the fact that, after more than 30 years after the discovery of its photoluminescence. To date, the PC thanks to its unusual properties is considered quite promising material of modern semiconductor electronics. Por-Si films may consist of a set of vertical wirelike structures. In this case, we can obtain the conditions of maximum absorption of photons in the porous film to increase efficiency of solar cells [2]. Detailed understanding of optical phenomena in nanofilm srequires some knowledge of physical foundations of electrical phenomena. Electrical properties of nanofilms can have direct practical applications. For example, porous silicon nanofilm can be used along with semiconductor diodes for generation of chaotic, broadband, high-frequency signals in electronic circuits.
Hierarchical structures of nanofilms create many potential barriers of the electric field. These are lot of well-known theoretical and experimental investigations of electron tunneling in multi-barrier structures - superlattices with defined regular forms ofdistribution of potential [10]. We take into account that process of tunneling is resonant: electrons pass through potential barrier with energies close to their own quantum energy levels of the barrier. If structure of the barriers not strictly ordered, for example, in case of fractal structure, the eigenvalues of the energy levels are unknown; the resonance effect is difficult to distinguish. It is necessary for estimation of intensity of transmission of electrons through the barrier.

So, study of process of tunneling of electrons in nanofilms with fractal cluster structure is an interesting research problem. Structure of potential barrier in such films is complex. The aim of this work is to study electrical properties of a porous silicon nanofilm.

\section{Experiment}

Thin films of porous silicon were obtained by electrochemical etching in electrolyte with ratio of $\mathrm{HF}: \mathrm{C}_{2} \mathrm{H}_{5} \mathrm{OH}-1: 1.5$ (fig.1).

\footnotetext{
* Corresponding author: e-mail:erulan.s@yandex.ru
} 


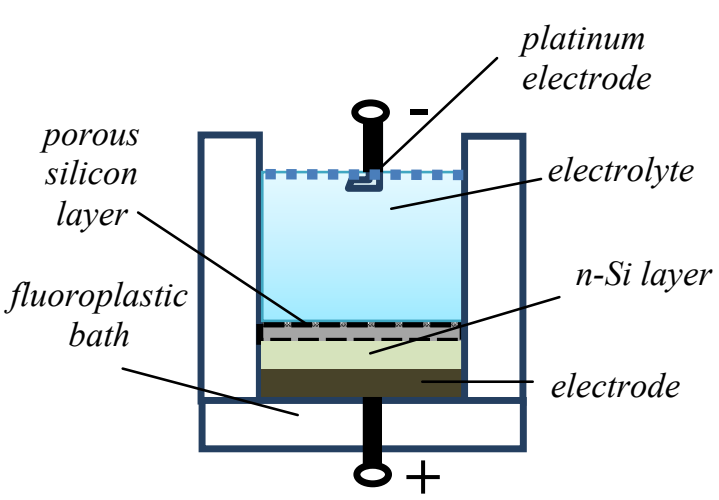

Figure 1 - Electrolytic cell forobtaining of thin films of porous silicon

Electrochemical etching has three modes: galvanostatic (constant current etching), potentiostatic (etching at a constant voltage), and the combined. The anode of a silicon wafer is placed in an electrochemical cell which is often made of polytetrafluorethylene (teflon). The electrolyte is a chemical liquid containing hydrofluoric acid (HF) and various organic compounds. As a cathode serves material resistant to hydrofluoric acid (platinum, ultra-pure silicon).Geometric shapes and pore sizes, porosity and some physical properties depend on the etching parameters and the electrolyte composition, the type of semiconductor, the doping level[11,12].

For obtaining most degree of porosity and uniform distribution of porosity we use $n$-type silicon, wherein concentration of electrons was $10^{19} \mathrm{~cm}^{-3}$.

Photo of morphology of the porous film obtained by scanning probe microscopy is strongly inhomogeneous. Wirelike peaks on the surface of the film have thickness about $100 \mathrm{~nm}$. Thickness of the films depends on etching time. For simplicity of electrical measures we choose etching time about $5 s$. In this case, maximum length of the wirelike peaks reaches about 0,5 micrometers.

Metal contacts deposited to $n$-layer and poroussurface (fig.2) provide the specific current-voltage characteristic of porous silicon nanofilms. Voltage appliedtothe film was in range from $-7 V$ to $7 V$ with step $0,9 \mathrm{~V}$. Source of energy was the Two-Wire Current-Voltage Analyzer of the universal station NI ELVIS II ${ }^{+}$, where current intensity measured in $m A$.

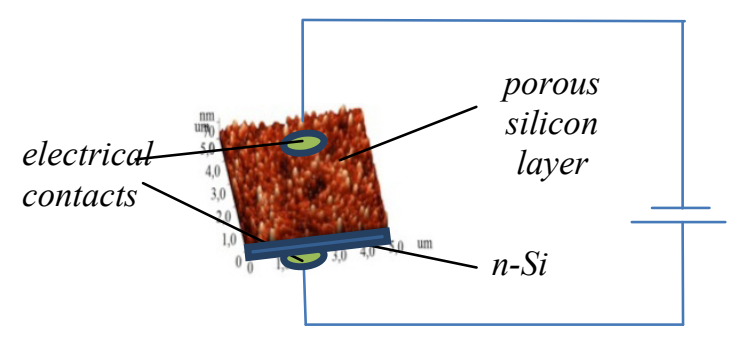

Figure 2 - Scheme of contacts for the $C-V$ characteristics of porous silicon

\section{Results and Discussion}

Figure 3 shows dependence of variations of current $I$ through a set of silicon nanofilms on voltage $V$, applied into opposite surfaces of the thin film.

Current-voltage characteristic depends on method of application of increasing or decreasing. At current greater than $0.5 \mathrm{~mA}$, structure and properties of the nanofilmswere changed. It will be seen in figure 3, corresponding to applying voltage from $7 \mathrm{~V}$ to $+7 \mathrm{~V}$ with step $0.9 \mathrm{~V}$. Such regularity is observed in repeated measurements (number of measurements is 10). After the establishment of equilibrium temperature initial electrical properties of nanofilms can be restored, but some time later the electrical properties can change again because of oxidation. Another feature of current-voltage characteristic of nanofilms is decreasing of current with increasing of voltage in several local areas (fig.3). This effect can be described by introducing the concept of negative resistance; its physical meaning is in resonant tunneling of electrons through a potential barrier.

In contrast to the results of modern research of nanostructures [13] silicon nanofilms contains several potential barriers. Because of this oscillations are observed in current-voltage characteristic. Typically, a multi-barrier tunneling of electrons observed in heterojunctions.

Multi-barrier structure ofporous silicon nanofilms disappears after passing of current with relatively big value considerable strength, as their structure is somewhat leveled. It can be seen in figure 3, as the oscillation peaks are smoothed. 


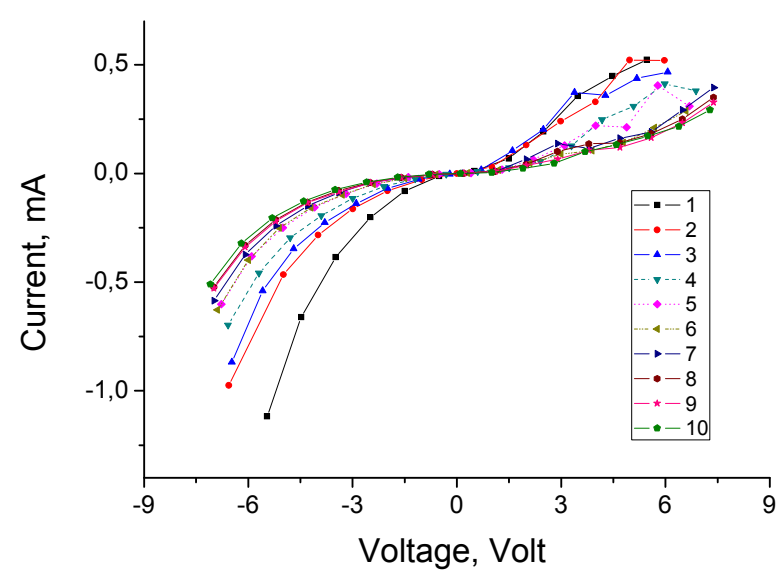

Figure 3 - Current-voltage characteristics of porous silicon in the direction of InGa -nSi-porSi-InGa

This result means that potential barriers in porous silicon nanofilms are formed not by contact transitions, but by non-equilibrium distribution of charge carriers in a nanofilm. Indeed, if we shall consider current at $U<0$ (Fig. 3) as reverse current, it is not small as in $p / n$ junction. On the contrary, $I$ $(U<0)>I(U>0)$. Some asymmetry at $U>0, U<$ 0 shown in figure 4 , probably explained by the fact that the voltage applied is $U<0$ to $n$-layer, here concentration of electrons more than in the other contact of nanofilm repels electrons and contributes to increasing of value of current.

Multi-Barrier resonant tunneling effects are more clear if current-voltage characteristic representsin relative variables $I / I_{l}, U / U_{l}$, where $I_{l}, U_{l}$ are current and voltage corresponding to first maximum of the curve $I=I(V)$ (fig.5).

Using of the values $I_{l}, U_{l}$ let us also estimate an absolute value of as differential resistance $R=R_{l}$ in some characteristic point with $\mathrm{I}_{1}$ and $\mathrm{U}_{1}$ :

$$
R_{1}^{-1}=\lim _{\Delta U \rightarrow 0}\left|\frac{\Delta I}{\Delta U}\right|_{U_{1}}=\frac{I_{1}}{U_{1}} .
$$

Figure 6 shows strongly nonlinear dependence of $R_{I}=R_{l}\left(I_{1}\right)$ : electrical resistance of nanofilms strongly depends on current through them.

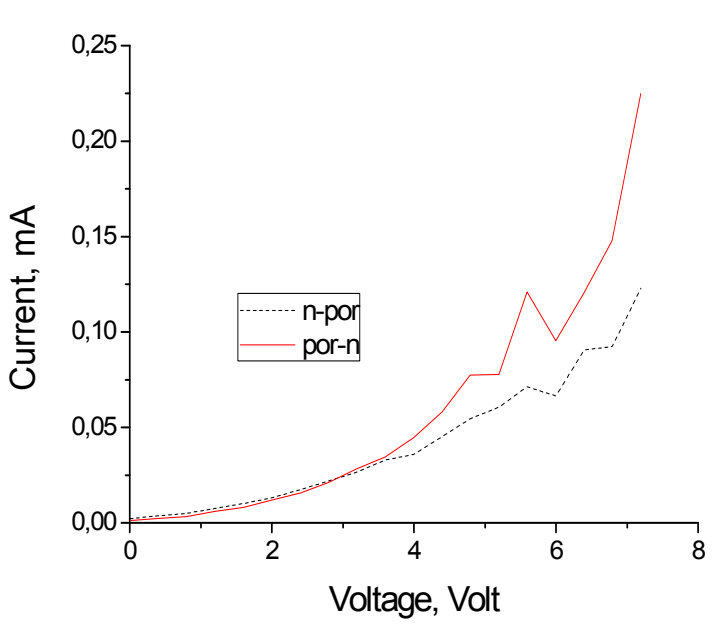

Figure $4-\mathrm{CVC}$ at different polarity from $0 \mathrm{~V}$ to $7 \mathrm{~V}$

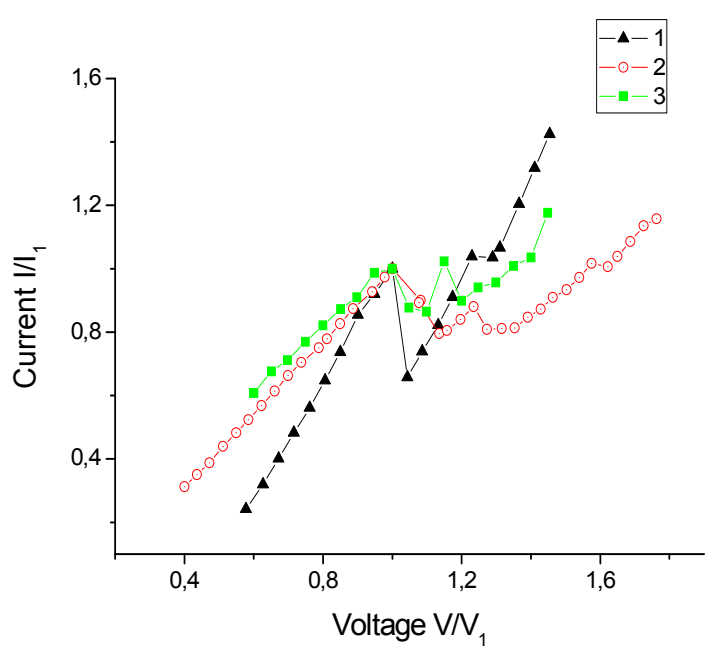

Figure 5 - The current-voltage characteristic in relative variables. $I 1, V 1$ - current and voltage corresponding to the first peak. $V_{l}: 1-1,706 \mathrm{~V}$, 2-2,077V, 3-1,515V; $I_{1}: 1-13,765 m A$, 2-6,267mA, 3-0,029mA. 


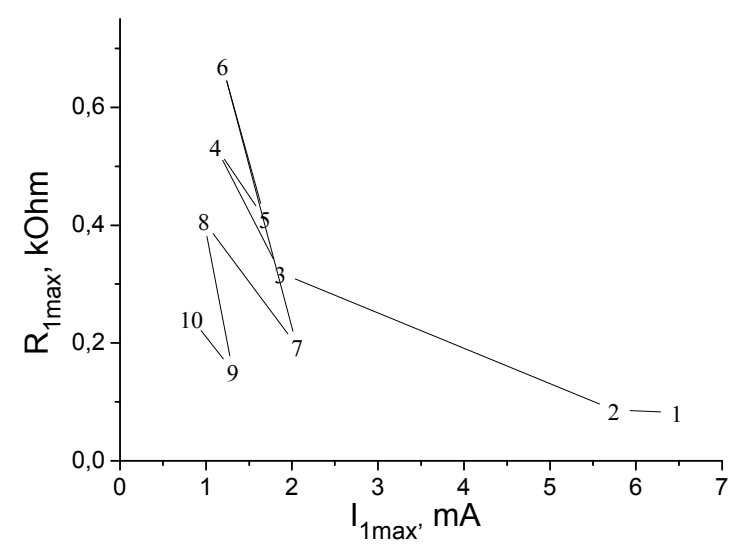

Figure 6-Change of the absolute value of differential resistance ofnanofilm at the first peak of current. Numbers in the figure correspond to number of measurement of current flowing through a film.

\section{Discussion}

Let us discuss possible explanations of the experimentally obtained new effects based on universal physical regularities.

Presence of multiple potential barriers in nanoscaleporous silicon films can be explained by their inhomogeneous, cluster structure. Recent experiments of $V$.Sivakov et al [14] show the presence of internal structures of nanofilms. These structures have different spatial scales with inhomogeneous distribution of electrons. The structures of all sizes and geometric shapes create different potential barriers for electrons moving. In quantum-sized nanostructures electrons can take discrete values of energy, therefore, Multi-Barrier resonant tunneling effects can be realized.

The strong dependence of electrical resistance of nanofilms on current is the demonstration of general properties of nonlinear objects whose properties depend on the processes occurring in them. Structure of the nanofilms, as well as surface morphology of the film has a hierarchical (fractal) structure. In principle, even arbitrarily weak current can cause a change in the small-scale structure of nanofilms. A quantitative description of strongly nonlinear phenomena is possible by using the concept of nonlinear fractal, whose minimal size of discrete structures depends on magnitude of measured fractal measure $[15,16]$.

\section{Conclusion}

Multi-barrier resonant tunneling of electrons in nanoscale wirelike structures of homogeneous material (silicon) can significantly enhance ability of existing technologies of nanoelectronics based on the use of heterojunctions. Results of the work let usadequately describe optical phenomena in solar cells with antireflection porous surface.Existence of negative differential resistance of nanofilms in a wide range of electron energy allows to realize broadband chaos generators operating on high frequencies.

\section{References}

[1] Föll H., Christophersen M., Carstensen J. \&Hasse G. Formation and application of porous silicon// Materials Science and Engineering. R.- 2002.- Vol. 280.- pp. 1-49. [2] Cullis A.G., Canham L.T. \&Calcott P.D.J. The structural and luminescence properties of porous silicon// J App Phys.-1997. - Vol 82.- No 3.- pp 909-965.

[3] Canham, L, Editor, Properties of porous silicon, INSPEC - The Institution of Electrical Engineers, 1997, United Kingdom.

[4] Parkhutik V. Porous silicon - mechanism of growth and applications.Solid-state Electron.1999, Vol.43, pp. 1121-1141.

[5] Vasquez, RP.,Fathauer, RW., George, T., Ksendzov, A. \& Lin, TL. Electronic structure of light emitting porous Si, ApplPhyLett, 1992, Vol. 60, No. 8, pp. 10041006.

[6] Beale, M. I. J., Benjamin, J. D., Uren, M. J., Chew, N. G. \&Cullis, A. G. The formation of porous silicon by chemical stain etches, J. Crys. Growth, 1986, Vol. 75, pp. 408.

[7] Zubko, V.G., Smith, T.L. \& Witt, A.N. Silicon Nanoparticles and Interstellar Extinction, The Astrophysical Journal Letters, 1999, Vol. 511, pp. L57.

[8] G. Algun, M.C. Arikan, An Investigation of Electrical Properties of Porous Silicon, Tr. J. of Physics, 1999, Vol. 23, 789 - 797.

[9] A. Uhlir, Jr., Electrolytic Shaping of Germanium and Silicon, The Bell System TechnicalJournal, March, 1956,Vol. 35(2), 333-347.

[10] Britnell L. et al. Resonant tunnelling and negative differential conductance in graphene transistors// Nat. Commun. - 2013.4:1794 doi: 10.1038/ncomms2817. pp. 1-5.

[11] F. A. Ben Hander, J. D. Moreno, M. L. Marcos and J. González Velasco, Electrochemical Behaviour of Porous Silicon Layers Prepared byStain Etching Processes, 
Journal of New Materials for Electrochemical Systems,2003, Vol.6, 129-135.

[12] Husnen R. Abd, Y. Al-Douri, Naser M. Ahmed, U. Hashim, Alternative-Current Electrochemical Etching of Uniform Porous Silicon for Photodetector Applications, International Journal ofElectrochemicalScience, 2013, Vol.8, 11461 - 11473.

[13] MaoXiang Wang, JianHua Yu1, ChengXiu Sun. Light emission characteristics and negative resistance phenomenon of Si-based metal/insulator/semiconductor tunnel junction// Applied Surface Science.- 2000.- Vol. 161.- Issues 1-2. 1.- pp. 9-13.
[14] SivakovV., Voigt F., Hoffmann B., et al. Wet Chemically Etched Silicon Nanowire Architectures: Formation and Properties// Nanowires-Fundamental Research.InTech.-2011.- pp.45-80.

[15] ZhanabaevZ.Zh., GrevtsevaT.Yu. Physical Fractal Phenomena in Nanostructured Semiconductors// Reviews in Theoretical Science. - 2014. - Vol. 2.-No 3. - pp.211259.

[16] ZhanabaevZ. Zh., KozhagulovE.T. A generic model for scale-invariant neural networks// Journal of Neuroscience and Neuroengineering.- 2013.- Vol.2. - No.3 - P. 267-271. 